\title{
Electronic structure of $\mathrm{GaAs}(001)$
}

\author{
J. Olde, G. Mante, H.-P. Barnscheidt, L. Kipp, J.-C. Kuhr, \\ R. Manzke, and M. Skibowski \\ Institut für Experimentalphysik, Universität Kiel, Olshausenstrasse 40, \\ D-2300 Kiel 1, Federal Republic of Germany \\ J. Henk and W. Schattke \\ Institut für Theoretische Physik und Sternwarte, Universität Kiel, Olshausenstrasse 40, \\ D-2300 Kiel 1, Federal Republic of Germany \\ (Received 5 December 1989)
}

\begin{abstract}
The bulk valence-band structure along the $\Gamma X$ line of the GaAs(001)-c $(4 \times 4)$ surface prepared by molecular-beam epitaxy is investigated by angle-resolved photoemission using $\mathrm{He} \mathrm{I}$ and synchrotron radiation with photon energies between 10 and $34 \mathrm{eV}$. In order to consider explicitly the final-state effects, the technique of structure plots is applied using free-electron-like final states as well as complete pseudopotential calculations for the unoccupied bands. In this way nearly all experimentally observed peaks can be consistently explained, except two peaks close to the valence-band maximum, which have to be attributed to surface states.
\end{abstract}

\section{INTRODUCTION}

With its increasing technological importance GaAs has become an interesting material in basic research, including transport and electronic properties as well as surface and interface structure. Up to now, experimentally and theoretically the (110) surface is the best-studied plane of GaAs. This is because smooth and clean surfaces can be prepared simply by cleaving bulk crystals under ultrahigh-vacuum (UHV) conditions and the surface atoms react only by relaxation instead of more complicated surface reconstructions.

For electronic devices, however, the (001) plane is usually applied. This plane is not cleavable and molecularbeam epitaxy (MBE) has to be applied for preparation of good surfaces. In addition, depending on the particular preparation conditions, the (001) surface exhibits several geometrical reconstructions, see, e.g., Refs. 1-3. Thus, only few experimental investigations, concerning the electronic structure of the surface and of the bulk along the $\Delta$ line by means of angle-resolved photoemission spectroscopy (ARPES), have been published for this plane. ${ }^{4-9}$ Especially for the interpretation of the observed bulk features in normal emission, only the main branch of a free-electron parabola normal to the surface has been considered for the final state. ${ }^{8,9}$ This way, not all of the bulk emissions could be explained, especially at lower photon energies. $^{9}$

The aim of the present work is to reinvestigate the experimental band structure of $\mathrm{GaAs}(001)$ from normalemission spectra in a detailed analysis and to compare the results with theoretical bulk band structures including a thorough discussion of final-state effects. It will be shown that the technique of structure plots is a good tool to explain the experimental data for the (001) plane. The binding energies and dispersions of the main peaks can be described by transitions from the valence bands into backfolded free-electron-like final states, if a proper value of the inner potential is chosen. An even better understanding is achieved by using unoccupied states of a complete pseudopotential calculation. This gives new insight into the accuracy of the valence- and conduction-band calculations and their experimental determination from angle-resolved photoemission.

\section{EXPERIMENTAL}

The GaAs(001)-c $(4 \times 4)$ surfaces were prepared in our laboratory in Kiel with a MBE system equipped with two evaporation sources, one for gallium and one for arsenic $\left(\mathrm{As}_{4}\right)$. The samples, cut from a polished [001]-oriented wafer, were degreased and attached to the sample holder with liquid indium. They were cleaned in UHV with 800-eV argon ions for several hours. Heating of the sample at about $500^{\circ} \mathrm{C}$ for $2 \mathrm{~min}$ immediately before starting the growth process, restored the crystallinity of the surface. The growth was performed at a substrate temperature of $535^{\circ} \mathrm{C}$ and an $\mathrm{As}_{4}$ to $\mathrm{Ga}$ flux ratio of about 6:1 with a growth rate of about $1000 \AA / h$. At these conditions a $(2 \times 4)$ surface reconstruction occurred during the growth process. The reflection-high-energy electron diffraction (RHEED) patterns taken in situ at $5 \mathrm{keV}$ electron energy in [110] and [110] azimuths are shown in Figs. 1(a) and 1(b).

After $2 \mathrm{~h}$ the MBE process was stopped by closing the shutters of the evaporation sources and turning off the furnace heaters. In order to avoid additional As adsorption at the surface, the samples were cooled down to $300^{\circ} \mathrm{C}$ and held at this temperature until the As pressure had decreased into the $10^{-8}$ torr range. Then the sample heater was turned off and the samples cooled down to room temperature. Figure 1(c) shows the low-energy 
electron diffraction (LEED) pattern of the resulting $c(4 \times 4)$ surface at $116 \mathrm{eV}$ kinetic energy.

By use of magnetically driven rods, the samples were directly transferred into the spectrometer chamber equipped for angle-resolved photoemission spectroscopy with a rare-gas discharge lamp (He I). In order to investigate the samples also with tunable photon energies, a transportable UHV chamber with a battery-driven ion pump was designed that enabled the sample transport from Kiel to HASYLAB (Hamburg Synchrotron Radia-
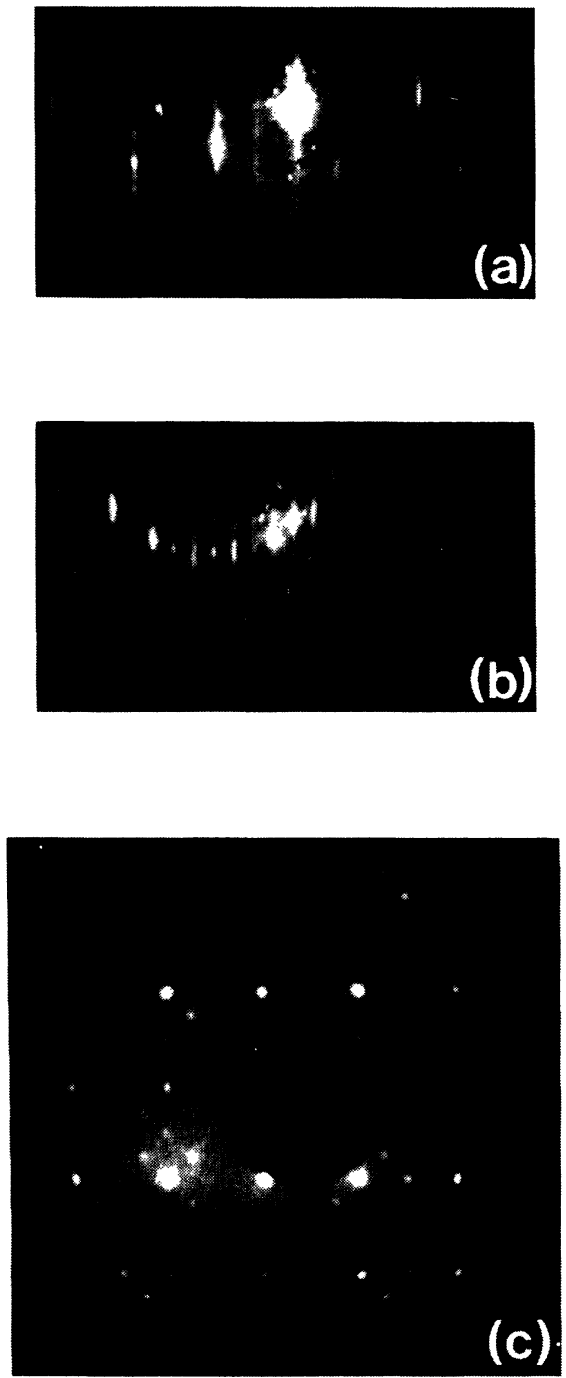

FIG. 1. (a) and (b) RHEED pattern of GaAs $(001)-(2 \times 4)$ during the growth at $535^{\circ} \mathrm{C}$ taken with $5 \mathrm{keV}$ electron energy. In (a), the [110] azimuth is shown. The periodicity is doubled relative to the ideal surface along [ 110$]$, i.e., every second streak is due to the $(1 \times 1)$ surface. (b) is the same for the [110] azimuth: The periodicity is four times that of the ideal $(1 \times 1)$ surface along [110]. The center of the pictures is overexposed due to imperfections in the screen. (c) LEED pattern of $\mathrm{GaAs}(001)$ $c(4 \times 4)$ at room temperature taken with $116 \mathrm{eV}$ electron energy. tion Laboratory) without any loss in surface quality. This was proven by comparing spectra taken with $\mathrm{He}$ I-lamp radiation under identical conditions in $\mathrm{Kiel}$ and in $\mathrm{Ham}$ burg. Both ARPES systems are equipped with a $180^{\circ}$ spherical analyzer mounted on a two-axis goniometer; the angular resolution is $\pm 0.5^{\circ}$ for polar and azimuthal angle and the energy resolution was chosen as $130 \mathrm{meV}$. The synchrotron radiation was varied in the photon-energy range from 10 to $34 \mathrm{eV}$ with a wavelength resolution of $2.3 \AA$.

\section{RESULTS AND DISCUSSION}

Angle-resolved photoemission spectra of $\mathrm{GaAs}(001)-$ $c(4 \times 4)$ taken in normal emission along the highsymmetry direction $\Gamma-\Delta-X$ (see Fig. 2 ) with photon energies in the range from 10 to $34 \mathrm{eV}$ are shown in Fig. 3. One observes six prominent structures that can be traced for a wide range of photon energies. The features $a, b$, and $c$ show clear dispersion and are therefore attributed

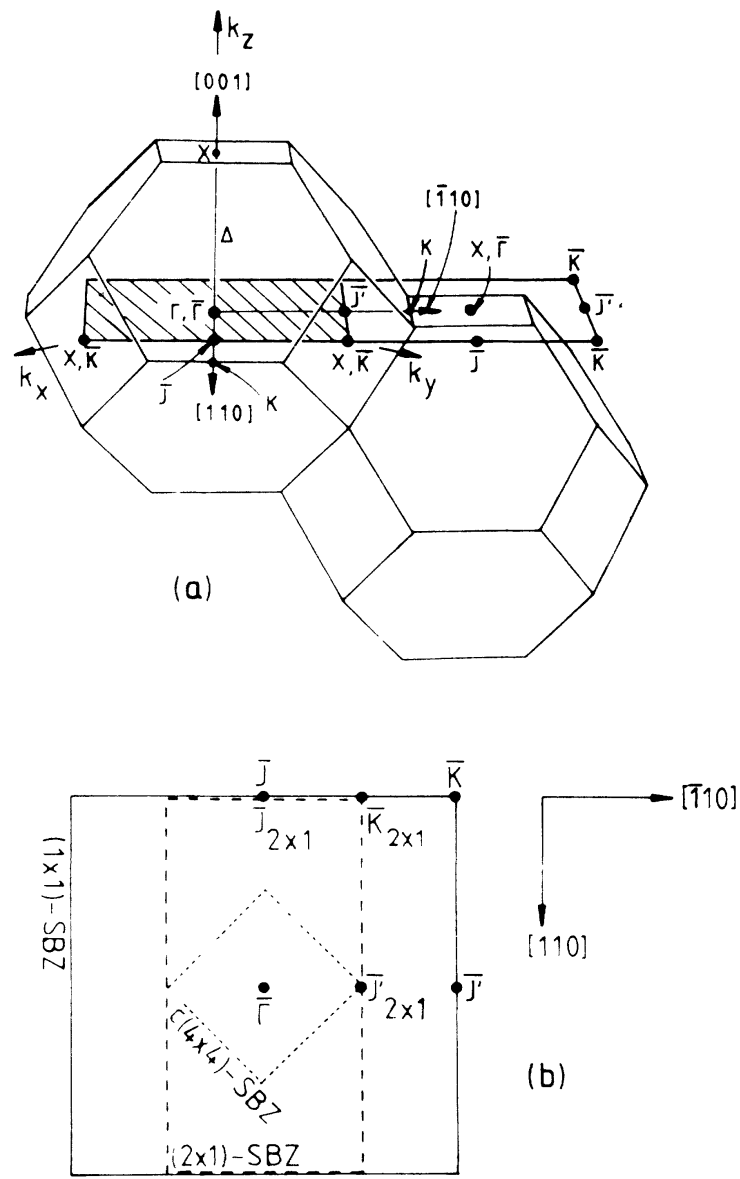

FIG. 2. (a) $(1 \times 1)$-surface Brillouin zone (SBZ) inserted into the bulk Brillouin zone of GaAs. In the [110] directions the periodicity of the $(1 \times 1) \mathrm{SBZ}$ is doubled compared to the bulk Brillouin zone. (b) Top view of the $(1 \times 1),(2 \times 1)$, and $c(4 \times 4)$ surface Brillouin zones. 
to transitions from bulk-derived states. Structures $S, S^{\prime}$, and $d$ reveal no dispersion with photon energy (momentum perpendicular to the surface) and may be due to emissions from surface states. In the following we will show that, nevertheless, feature $d$ is mainly caused by bulk effects, while $S$ and $S^{\prime}$ seem to be true surface states or resonances.

\section{A. Interpretation of photoemission spectra using free-electron-like final states}

Assuming free-electron-like final states and direct transitions an experimental valence-band structure can be obtained from the peaks in the normal-emission spectra by use of the well-known equation

$$
k_{\perp}=\left[\left(2 m / \hbar^{2}\right)\left(E_{\mathrm{kin}}+\left|V_{0}\right|\right)-\mathbf{g}_{\|}^{2}\right]^{1 / 2}-G_{\perp},
$$

where $k_{\perp}$ is the wave-vector component of electrons perpendicular to the surface, $E_{\text {kin }}$ the kinetic energy, $\left|V_{0}\right|$ the inner potential referred to the vacuum level, and $G_{\perp}$ the normal component of a bulk reciprocal-lattice vector $\mathbf{G}=\mathbf{G}_{1}+\mathbf{G}_{\|} \cdot \mathbf{g}_{\|}$is in general a linear combination of the parallel component of the reciprocal bulk lattice vector $\mathbf{G}$

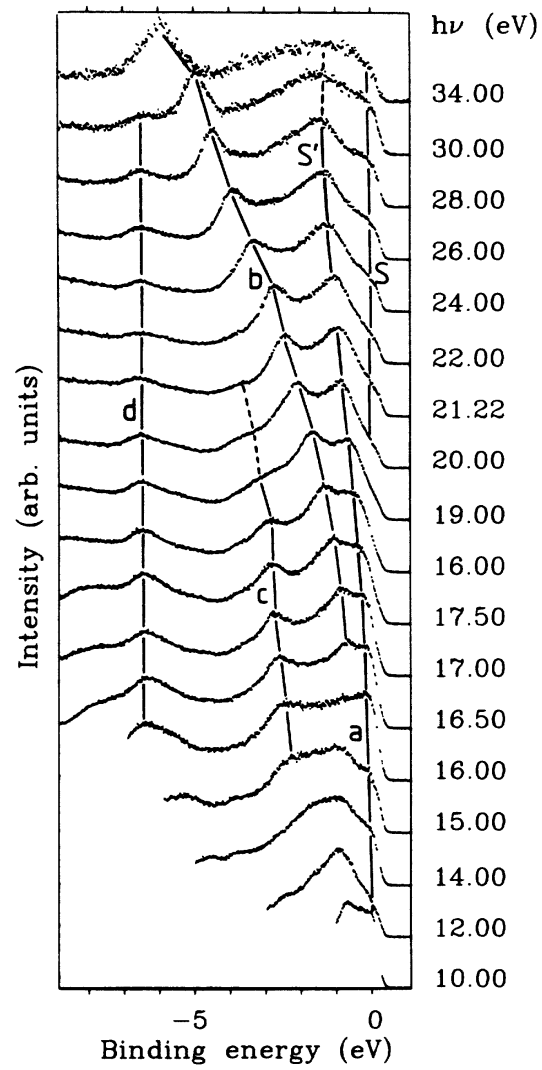

FIG. 3. Angle-resolved photoemission spectra taken in normal emission for different photon energies. The binding energy is related to the valence-band maximum (VBM). For the determination of the VBM, see text. and a reciprocal-lattice vector of the ideal or the reconstructed surface. The latter case should be also considered if the reciprocal surface lattice of $\mathrm{GaAs}(001)$ does not have the same periodicity as the bulk (see Fig. 2). The final-state energy $E_{f}$ referred to the valence-band maximum (VBM) for normal emission in accordance with Eq. (1a) is given by

$$
\begin{array}{r}
E_{f}=E_{\text {kin }}+U_{\text {th }}=\left(\hbar^{2} / 2 m\right)\left[\left(k_{\perp}+G_{\perp}\right)^{2}+\mathbf{g}_{\|}^{2}\right]-\left|E_{0}\right| \\
\text { with }\left|E_{0}\right|=\left|V_{0}\right|-U_{\text {th }},
\end{array}
$$

where $U_{\text {th }}$ is the photoelectron threshold.

An experimental valence-band structure obtained by Eq. (1a) is shown in Fig. 4, where we have considered only a single final-state band with $\left|V_{0}\right|=12 \mathrm{eV}, \mathrm{g}_{\|}=0$, and $G_{1}=4 \pi / a$; a is the lattice constant of GaAs. The solid lines labeled 1-3 show for comparison the theoretical valence-band structure calculated by Chelikowsky and Cohen. ${ }^{10}$ The VBM is attributed to the maximum kinetic energy of peak $a$. The value of $12 \mathrm{eV}$ is chosen for $\left|V_{0}\right|$ in order to get the best fit between the dispersion of peak $b$ and the calculated valence band 3 . This value is significantly lower than that given for $\operatorname{GaAs}(110){ }^{11}$

Peak $a$, showing up only for small values of $k_{\perp}$ up to about $\frac{1}{3} \mathbf{k}_{\Gamma X}$, lies close to the theoretical bands 1 and 2 . Deviations between the experimental points and the theory near VBM are associated with additional emission from state $S$ in this energy range, which cannot be clearly separated and causes possibly a fictitious shift of peak a to lower binding energies. For larger values of $k_{1}$ there are some additional weak emissions around $-2.5 \mathrm{eV}$, which can also be related to the uppermost bulk valence

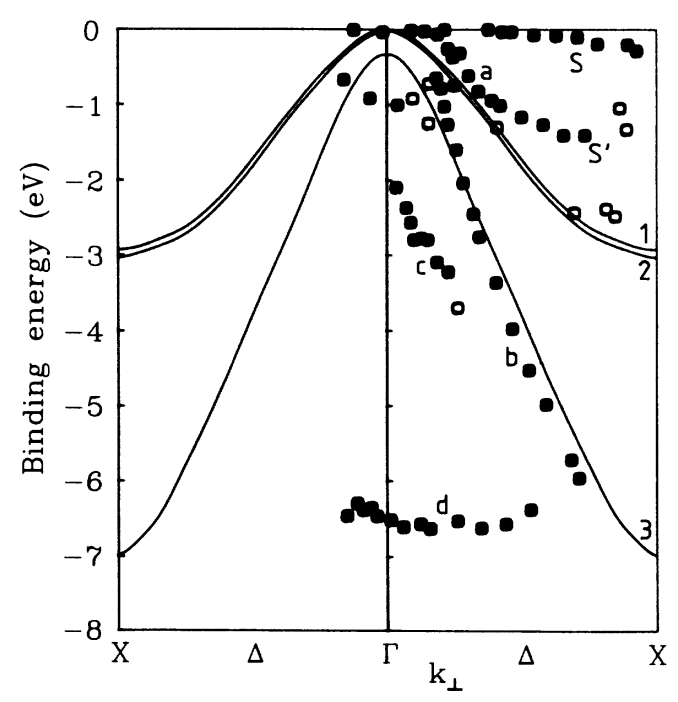

FIG. 4. Experimental bulk band structure along the direction $\Gamma X$ of GaAs by applying Eq. (1a). Solid circles indicate strong structures, open circles weaker structures. The lines show the theoretical band structure from Chelikowsky and Cohen (Ref. 10). 
bands. The distinct features $S, S^{\prime}$, and $d$ are far away from the theoretical bulk bands. $S$ and $S^{\prime}$ exhibit almost no dispersion with $k_{\perp}$ and therefore can be due to surface states or surface resonances as discussed later. An interpretation of $d$ is more difficult, because its energy is close to the critical point $X_{3}$ which has an energy of $-6.9 \mathrm{eV}$ following the calculation of Chelikowsky and Cohen ${ }^{10}$ or $-6.6 \mathrm{eV}$ following Cardona et al. ${ }^{12}$ Structure $c$ lies also distinctly apart from the theoretical bulk bands but exhibits strong dispersion and therefore, it must be due to emissions from bulk bands. Peak $c$ can be explained by transitions from the valence bands 1 or 2 into final states described by Eq. (1b) with a reciprocal-lattice vector $\mathbf{G}$ with nonvanishing parallel component (see further). The peaks at about $-1 \mathrm{eV}$ around point $\Gamma$ cannot be explained in the picture leading to Eqs. (1).

In order to achieve a more systematic analysis of finalstate effects, we now turn to the method of structure plots used previously by Williams et al. ${ }^{13}$ for the interpretation of the (110) surfaces of III-V compound semiconductors. This concept, which is not necessarily restricted to freeelectron-like final states, has proved to be very useful for the detailed analysis of normal-emission spectra. ${ }^{14}$ In Fig. 5, the basic idea of the method is shown: From a given theoretical band structure, a pair comprised of a valence and a conduction band is chosen [Fig. 5(a)]. Then for all values of $k_{\perp}$ of the Brillouin zone, the transition energies between the valence and the conduction band for $\mathbf{k}$-conserving transitions are determined, e.g., in Fig. 5(a) an excitation is possible for $k_{\perp A}$ for a photon en-
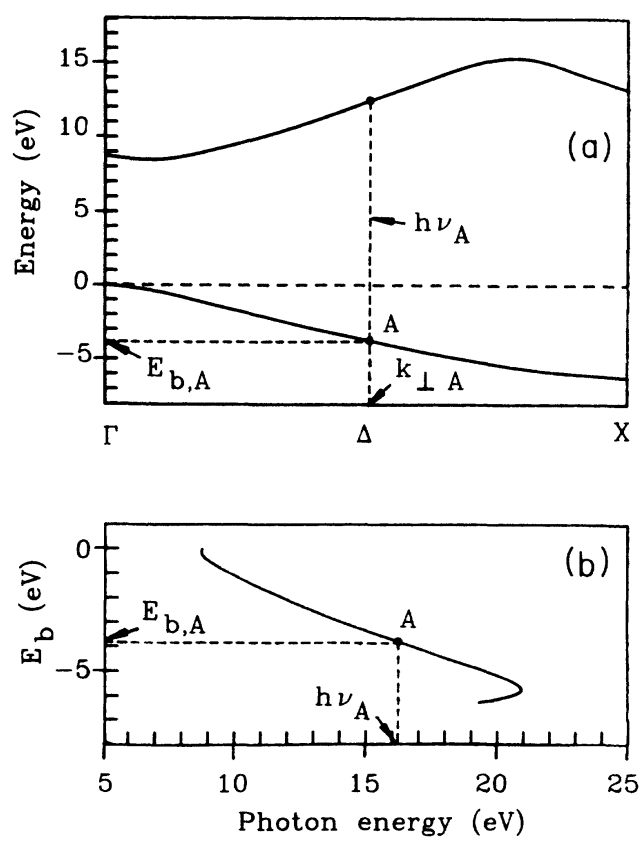

FIG. 5. Concept of a structure plot: (a) possible transition at $\boldsymbol{A}$ between one initial- and one final-state band; (b) structure plot resulting from all transitions of (a). $E_{b}$ is the binding energy relative to the VBM. ergy $h v_{A}$ at the initial-state energy $E_{b, A}$. These values are plotted into an $E_{b}(h v)$ diagram as shown in Fig. 5(b), resulting in a curve for the chosen pair of valence and conduction bands. Proceeding similarly for all other pairs of occupied and unoccupied bands, a collection of curves like that in Fig. 5(b) is obtained, called a structure plot, and it can be directly compared with experimental results without further assumptions.

For a first analysis we use in this subsection a valenceband structure calculated by the empirical tight-binding method and fitted to that of Chelikowsky and Cohen ${ }^{10}$ (for details see further) for the initial states and the freeelectron parabola given in Eq. (1b) for the final states. A refined analysis with a complete band-structure calculation will be given in Sec. III B. In accordance with the discussion above $\left|V_{0}\right|$ is taken as $12 \mathrm{eV}$. With a photoemission threshold $U_{\text {th }}=5.4 \mathrm{eV}$ for $\mathrm{GaAs}(001)$ (as an average from different values in Ref. 15) we have $\left|E_{0}\right|=6.6 \mathrm{eV}$. In Fig. 6 the resulting final-state band structure is shown. Here we considered those reciprocal bulk lattice vectors $\mathbf{G}$ resulting in bands, which can be reached in energy by transitions from the valence bands using our experimental photon energies [i.e., in Eq. (1b) only such pairs $G_{\perp}, g_{\|}$are used, for which the vector $\mathbf{G}_{\perp}+\mathbf{g}_{\|}$is a reciprocal bulk lattice vector]. Each band is labeled by a number from 4 to 13 corresponding to the reciprocal-lattice vectors given in Table $I$. Here [ijk] denote the components of $\mathbf{G}$ along $k_{x}, k_{y}$, and $k_{z}$ (see

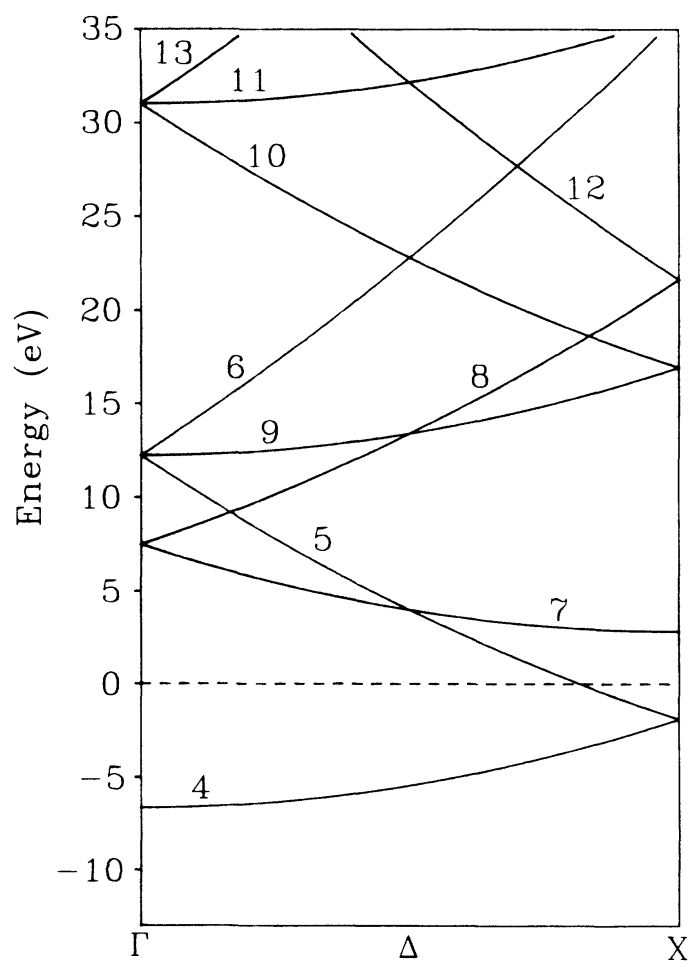

FIG. 6. Hypothetical band structure of GaAs along $\Gamma X$ in the free-electron parabola approximation after Eq. (1b) with $\left|V_{0}\right|=12 \mathrm{eV}$. The assignments are the same as in Table I. 
TABLE I. Reciprocal bulk lattice vectors $\mathbf{G}$ of GaAs used for backfolding of the free-electron final-state parabola into the first bulk Brillouin zone. In the first column the band index used in Fig. 6 is shown, in the second column the vectors are given in components of $k_{x}, k_{y}$, and $k_{z}$ (see Fig. 2), and in the third and fourth columns the components of $\mathbf{G}$ parallel and normal to the (001) surface are shown.

\begin{tabular}{|c|c|c|c|}
\hline $\begin{array}{l}\text { Final-state } \\
\text { index } \\
\text { (cf. Fig. 6) } \\
\end{array}$ & $\begin{array}{l}\text { Reciprocal-lattice } \\
\text { vector } \mathbf{G} \text { in } \\
\text { multiples of } 2 \pi / a\end{array}$ & $\begin{array}{l}\left|\mathbf{G}_{\|}\right| \text {in } \\
\text { multiples } \\
\text { of } 2 \pi / a\end{array}$ & $\begin{array}{c}G_{\perp} \text { in } \\
\text { multiples } \\
\text { of } 2 \pi / a \\
\end{array}$ \\
\hline 4 & {$[000]$} & 0 & 0 \\
\hline 5 & {$[00 \overline{2}]$} & 0 & -2 \\
\hline 6 & {$[002]$} & 0 & 2 \\
\hline 7 & 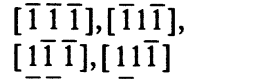 & $2^{1 / 2}$ & -1 \\
\hline 8 & $\begin{array}{l}{[\overline{1} 11],[\overline{1} 11],} \\
{[1 \overline{1} 1],[111]}\end{array}$ & $2^{1 / 2}$ & 1 \\
\hline 9 & $\begin{array}{l}{[\overline{2} 00],[0 \overline{2} 0]} \\
{[020],[200]}\end{array}$ & 2 & 0 \\
\hline 10 & $\begin{array}{l}{[\overline{2} 0 \overline{2}],[0 \overline{2} \overline{2}]} \\
{[02 \overline{2}],[\overline{2} 0 \overline{2}]}\end{array}$ & 2 & -2 \\
\hline 11 & $\begin{array}{l}{[\overline{2} \overline{2} 0],[\overline{2} 20]} \\
{[2 \overline{2} 0],[220]}\end{array}$ & $8^{1 / 2}$ & 0 \\
\hline 12 & 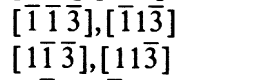 & $2^{1 / 2}$ & -3 \\
\hline 13 & $\begin{array}{l}{[0 \overline{2} 2],[\overline{2} 02]} \\
{[202],[022]}\end{array}$ & 2 & 2 \\
\hline
\end{tabular}
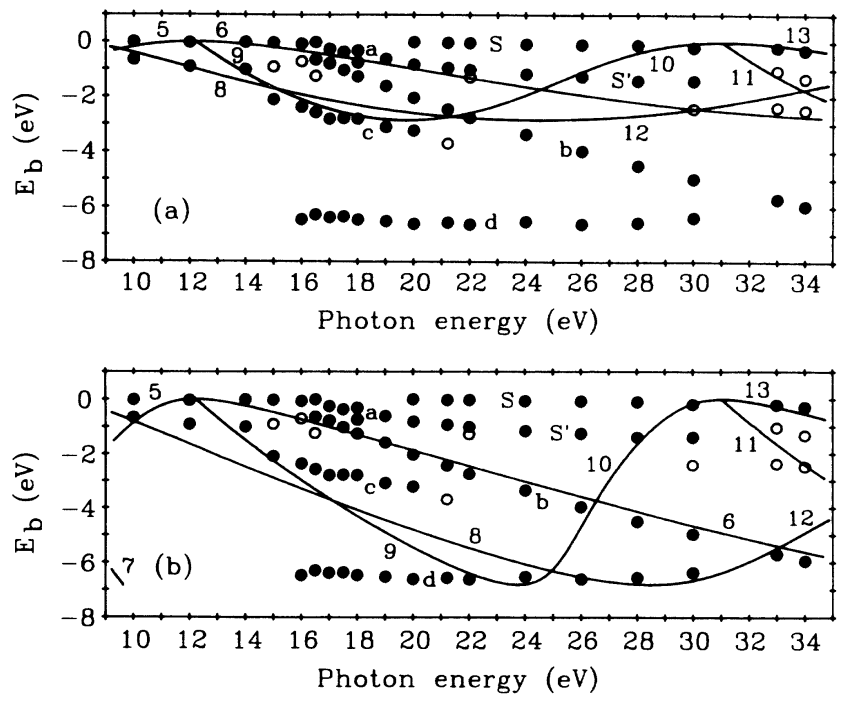

FIG. 7. Structure plots from the experimental data. Solid circles indicate strong structures, open circles weaker structures. The experimental transitions are assigned by letters similar to Fig. 3. (a) The solid lines are theoretical structure curves for transitions from initial state 1 (see Fig. 4) into possible freeelectron-like final states as shown in Fig. 6. Each curve has the same assignment as the final state in Fig. 6. (b) Same as (a), but for transitions from initial state 3 .
Fig. 2) in units of $2 \pi / a$, i.e., for example [ $\overline{1} 11]$ is the notation for $\mathbf{G}=(2 \pi / a)(-1,1,1)$.

The resulting structure plots are shown in Fig. 7. In Fig. 7(a) we used the uppermost valence band (labeled 1 in Fig. 4) as the initial state and in Fig. 7(b) the valence band 3 . The structure curves are numbered by the finalstate bands given in Fig. 6. Symmetry selection rules for the transitions have not been taken into account. The comparison with the included experimental results shows that the dispersing peaks $a$ and $b$ are well described by the theoretical curve 6 . This corresponds to the final state with $\mathbf{g}_{\|}=0$, which has been also used for the analysis in Fig. 4. This agreement is of course mainly due to the particular choice of the fit parameter $\left|V_{0}\right|$. The most striking fact here is the additional correspondence between peaks $c$ and curve 9 in Fig. 7(a). These peaks can be interpreted as due to emissions from valence band 1 or 2 into the final-state band described by all reciprocallattice vectors $G$ with $\left|G_{\|}\right|=4 \pi / a$ and $G_{1}=0$, which are the shortest reciprocal bulk lattice vectors parallel to the (001) surface. On the other hand, we find no theoretical curves lying close to the emissions of $S, S^{\prime}$, and $d$. Thus, within the free-electron-like final-state approximation and the concept of bulk interband transitions with $\mathbf{k}$ conservation, these peaks are not due to final-state effects.

According to the accuracy of the present analysis, it should be mentioned that for low-photon energies a unique explanation is somewhat problematical: Between 10 and $16 \mathrm{eV}$ photon energy some of the emission maxima around $-1 \mathrm{eV}$ binding energy can be related to curve 8 [Fig. 7(a)] and others to curves 5 and 6 [Fig. 7(b)]. In order to overcome these shortcomings we also tried final-state bands folded back by reciprocal-lattice vectors of the surface [i.e., $\mathbf{g}_{\|}$in Eq. (1b) is not only the parallel component of a bulk lattice vector], but without further success in the interpretation of the photoemission peaks in this photon-energy range. On the other hand, some theoretical curves are not confirmed by experiment, e.g., curve 8 in Fig. 7(b). This may be attributed to the inadequacy of the free-electron final-state band structure.

At this point, the following conclusions can be drawn: All peaks with strong dispersion in the normal-emission spectra can be consistently explained by combining the free-electron-like final-state parabola with the valenceband-structure calculations by Chelikowsky and Cohen, ${ }^{10}$ if different branches (i.e., backfolding with different reciprocal bulk lattice vectors) are considered. This also supports the accuracy of the valence-band-structure calculations. The small deviations between the theoretical and experimental curves may be due to the inaccuracies of the valence-band calculations or also a consequence of a principle problem by applying the model described earlier: The free-electron parabola is only a first approximation for the real final-state band structure. It is known from conduction-band calculations (see further) that the periodic potential of the crystal leads to band splittings and shifts of the backfolded parabola, which are different from band to band. This may result at least in an energy-dependent value of $\left|V_{0}\right|$ (see Ref. 9) degrading $\left|V_{0}\right|$ to a band-and energy-dependent fit parameter. 


\section{B. Interpretation of photoemission spectra using final states calculated by an empirical pseudopotential}

In order to improve the analysis of the experimental data we now turn to more realistic states derived from a complete band-structure calculation. Before the discussion of the structure plots the applied theoretical methods will be briefly introduced: We utilized the empirical tight-binding method (ETBM) for calculating the valence bands and the empirical pseudopotential method (EPM) for the conduction bands. The spin-orbit splitting is not included in the calculations. The main difference from other ETBM calculations of the energy bands of semiconductors is that we make use of the extended Hückel theory (EHT), ${ }^{16}$ which establishes a connection between the matrix elements of the Hamiltonian $H$ and the overlap matrix $S$ :

$$
\begin{aligned}
& H_{\alpha l \beta m}(\mathbf{k})=-\frac{1}{2} K_{\alpha \beta}\left(I_{\alpha l}+I_{\beta m}\right) S_{\alpha l \beta m}(\mathbf{k}) \\
& \quad \text { for }(\alpha l) \neq(\beta m), \\
& H_{\alpha l \alpha l}(\mathbf{k})=-\widetilde{I}_{\alpha l}-K_{\alpha \alpha} I_{\alpha l}\left[S_{\alpha l \alpha l}(\mathbf{k})-1\right] .
\end{aligned}
$$

Here $\alpha$ and $\beta$ denote the orbitals and $l$ and $m$ the basic atoms in the unit cell. The parameters $K_{\alpha \beta}, I_{\alpha l}$, and $\widetilde{I}_{\alpha l}$

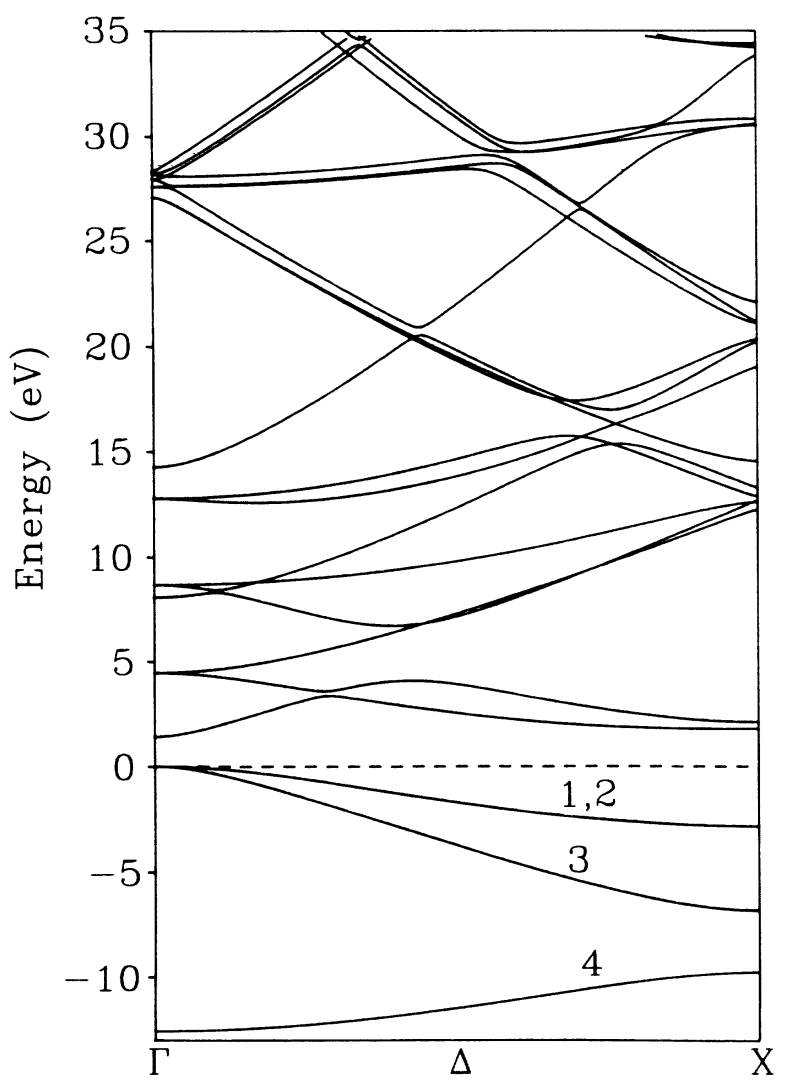

FIG. 8. Theoretical bulk band structure of GaAs along $\Gamma X$. The valence bands 1-4 are calculated by an empirical tightbinding method, the conduction bands by an empirical pseudopotential method. are determined by fitting the bulk band structure to the calculated one of Chelikowsky and Cohen. ${ }^{10}$ Thus the overlap integrals have to be calculated explicitly. Our basic wave functions consist of those calculated by Clementi and Roetti ${ }^{17}$ via the Roothaan-Hartree-Fock method. We considered all interactions up to thirdnearest-neighbor atoms. For the calculation of the conduction bands via the EPM we applied the local potential used by Cohen and Bergstresser. ${ }^{18} 137$ reciprocal-lattice vectors were taken into account, treating 51 exactly and the rest with Löwdin-type perturbation theory. The accuracy of this calculation was proved for $\mathrm{GaAs}(110)$ in Ref. 14. The resulting band structure is shown in Fig. 8.

To determine the origin of the bands calculated with the EPM, we have multiplied the local potential $V(r)$ with a factor $\alpha$, with $\alpha$ in the range from 0 to 1 . At $\alpha=0$ the potential vanishes and we get a free-electron parabola starting at $8.9 \mathrm{eV}$ below the VBM. For example, the branch belonging to $\mathbf{G}=(2 \pi / a)(0,0,2)$ begins to move upwards to higher energies for increasing $\alpha$. For $\alpha>0.5$ it mixes with other states, but the main parts can be described by a free-electron-like parabola branch with $\mathbf{G}=(2 \pi / a)(0,0,2)$ and increasing $\left|E_{0}\right|$ [see Eq. (1b)], up to about $6.5 \mathrm{eV}$ for $\alpha=1$.

The structure plots obtained from our band structure are given in Fig. 9 together with the experimental results. Symmetry selection rules are not taken into account. In Fig. 9(a), the uppermost valence bands 1 and 2 (which are commonly degenerate in this calculation) are used for the initial states; in Fig. 9(b) valence band 3 is used. As in the analysis with free-electron-like final states the peaks $b$ with strong $k_{\perp}$ dispersion are excellently described by theoretical curves in Fig. 9(b), but in contrast to the methods described earlier, one should mention that for the present analysis no fit parameter like $\left|V_{0}\right|$ is necessary in order to achieve this agreement between experiment
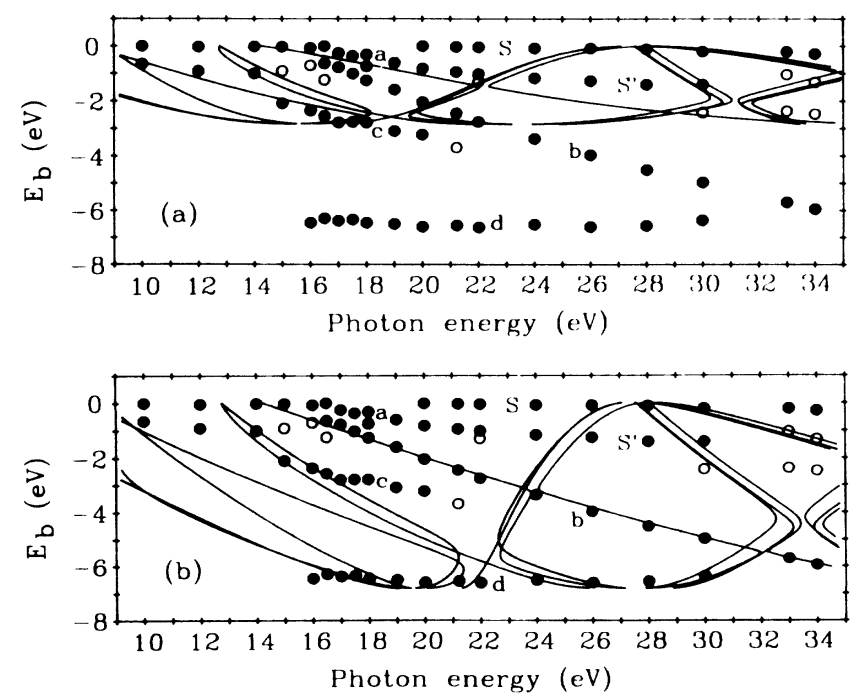

FIG. 9. Comparison of the experimental structure plots (like in Fig. 7) with calculated ones using the pseudopotential final states of Fig. 8. (a) From initial states 1 or 2. (b) From initial state 3 . 
and theory. Structure $a$ can be interpreted by transitions from valence band 1 or 2 into the same final state as the emissions of $b$. Here we remark that also in the EPM model the parts of theoretical curves coinciding with feature $b$ are mainly derived from $\mathbf{G}=(2 \pi / a)(0,0,2)$.

Feature $c$ is a distinct transition from valence band 1 or 2 into several final states lying relatively close to the bands 8 and 9 of the free-electron-like final states. The peaks at about $-1 \mathrm{eV}$ for lower photon energies may be explained by direct transitions into different final states similar to Fig. 7.

As a result of the multiple band splittings and shifts, which occur, if the free-electron final-state parabola is replaced by the pseudopotential conduction-band structure, we find a concentration of structure curves in the energy range about $-6.6 \mathrm{eV}$. Therefore $d$ can be explained by transitions from valence-band states near the $X_{3}$ critical point into several final states, in good agreement with the critical-point energy calculated in Ref. 12. Thus feature $d$ seems to be mainly bulk derived, although it reveals experimentally no significant $k_{\perp}$ dispersion, and final-state effects become very important in the explanation of this structure. In contrast to the free-electron-like final-state model, where these peaks were only explainable by density-of-states effects, here a very satisfying interpretation by considering only k-conserving transitions is given.

Peaks $S$ and $S^{\prime}$ are obviously surface states or resonances since the emissions of $S^{\prime}$ lie distinctly apart from theoretical curves and only some emissions of $S$ are lying on structure curves. In addition, we show below that $S$ reveals the periodicity of a reconstructed surface. Here we note a very recent EPM calculation of the conduction bands up to $20 \mathrm{eV}$ above VBM of Chelikowsky et al. ${ }^{19}$ In comparison with our calculations nonlocal corrections to the pseudopotential are considered. Structure plots obtained from these bands are very similar to ours of Fig. 9 for most structure curves, and within experimental accuracy no significant improvements between theory and experiment are achieved.

Similar to the analysis with free-electron-like final states, there are calculated bands that are not observed experimentally. This fact may result from symmetry selection rules and/or small transition matrix element effects. For a more refined analysis the coupling of the final states to the vacuum should be considered.

\section{Off-normal measurements}

Here we briefly summarize the results from off-normal measurements. In the spectra taken with different polar angles along the directions of high symmetry most peaks show only very weak dispersion and strong influences from the critical points $X_{5}, \Sigma_{1 \mathrm{~min}}$, and $X_{3}$ are observed. Only the states near the VBM exhibit stronger dispersion with $\mathbf{k}_{\|}$. Here we only discuss the results for the uppermost surface band $S$ along the directions $\bar{\Gamma} \bar{J}$ and $\bar{\Gamma} \bar{J}^{\prime}$ of the surface Brillouin zone referring to the ideal $(1 \times 1)$ surface (see Fig. 2). The observed emissions are shown in Fig. 10 in comparison with the results of Larsen et al. ${ }^{5}$ and Bringans and Bachrach. ${ }^{6}$ Here all data are normalized with respect to the energy of this surface state at the
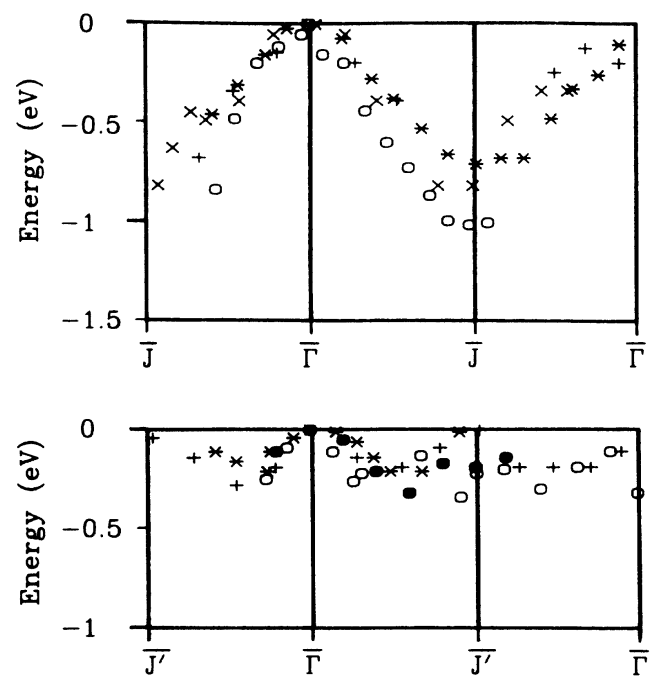

FIG. 10. Dispersion of the uppermost occupied surface derived band along the direction $\bar{\Gamma} \bar{J}$ and $\bar{\Gamma} \bar{J}^{\prime}$ [referred to the (1X1)-SBZ]. The energy is related to the surface-state maximum: $O$, our data at 21.22-eV photon energy; $O$, at $26 \mathrm{eV} ; \times$, data of Bringans and Bachrach (Ref. 6) at $20 \mathrm{eV}$; +, at $25 \mathrm{eV}$; *, data of Larsen et al. (Ref. 5) at $29 \mathrm{eV}$. For details see text.

first $\bar{\Gamma}$ point, i.e., the kinetic energy of the peaks in normal emission. From all measurements it can be seen, that $S$ shows the simple periodicity of the $(1 \times 1)$ surface along $\bar{\Gamma} \bar{J}$, while it seems to be doubled along $\bar{\Gamma} \bar{J}^{\prime}$.

The assignment of $S$ (and also $S^{\prime}$ ) to surface states or resonances is supported by theoretical band structures that are based on the assumption that the dominant ordering mechanism is the dimerization of the uppermost surface As atoms. Calculating the band structure of a (2×1)-reconstructed surface Larsen et al. ${ }^{4}$ find a mainly As-derived state near VBM that has only weak dispersion along $\bar{\Gamma} \bar{J}^{\prime}$ but disperses along $\bar{\Gamma} \bar{J}$ to higher-binding energies up to about $-1 \mathrm{eV}$ at $\bar{J}$. In principle, feature $S$ reveals this behavior. Another theoretical surface band at about $-1 \mathrm{eV}$ at $\bar{\Gamma}$ may be identified with $S^{\prime}$. Recent calculations $\mathrm{s}^{20}$ confirm the theoretical band structure of the $(2 \times 1)$ surface; the state at about $-1 \mathrm{eV}$ is found to be As backbond derived, similar to the state near the fundamental gap that contains strong admixtures of the As dangling bond. Here also the surface bands resulting from a $c(2 \times 2)$ and a $p(2 \times 2)$ reconstruction are calculated, but without achieving an overall better correspondence with the experimental data. However, even if $S$ shows not the periodicity expected for the $c(4 \times 4)$ surface evident from the LEED and RHEED measurements, it is clearly a surface state since its periodicity is different from that of the bulk. The remaining question, why the periodicity observed in LEED and RHEED is not the same as in photoemission experiments, may be clarified by future improvements of the surface preparation as well as band-structure calculations of the $c(4 \times 4)$ surface based on reliable surface models. 


\section{CONCLUSIONS}

We have studied the electronic structure of $\mathrm{GaAs}(001)$ by angle-resolved photoemission spectroscopy mainly in normal emission. All features in the spectra exhibiting significant dispersion can be explained by assuming freeelectron-like final states with an inner potential $\left|V_{0}\right|$ used as a fit parameter. For a detailed interpretation the concept of structure plots was successfully used for normal emission. Although the assumption of free-electron-like final states together with a fixed inner potential is a relative crude approximation, a reasonable agreement between experiment and theory was achieved with one value of $\left|V_{0}\right|$. This $\left|V_{0}\right|$ is significantly lower than the value used for GaAs(110). We observed no peaks associated with backfolding by reciprocal-lattice vectors of the reconstructed surface. Three nondispersing features were found in the experiment, not predicted by the directtransition model using free-electron-like final states.

In a second step, empirical pseudopotential calculations have been performed. This way the problem of uncertainties in $\left|V_{0}\right|$ was overcome, and also band splittings in the final states were included. In the resulting structure plots an improved agreement between theory and experiment is achieved. In contrast to the free-electron-like final states, one of the nondispersing features becomes explainable by $\mathbf{k}$-conserving transitions into different final states. The remaining two emissions with no $k_{1}$ dispersion are interpreted as surface-related structures in reasonable agreement with theory. Thus, all peak positions in the spectra were explained considering explicitly final-state effects. The overall good correspondence between experiment and theory illuminates the reliability of present bulk band-structure calculations.

\section{ACKNOWLEDGMENTS}

We would like to thank Dr. G. Karschnick (now at Korth-Merck, Kiel) for his engagement in the early phases of the MBE and photoemission project and Dr. R. L. Johnson and Dr. K. Ploog (Max-Planck-Institut für Festkörperforschung, Stuttgart) for advice in the first steps of the design of MBE sources. This work was supported by the Bundesministerium für Forschung und Technologie (Bonn, Germany), Project No. 05301 AAI, by the Kultusministerium des Landes Schleswig-Holstein, Federal Republic of Germany, and by the Deutsche Forschungsgemeinschaft (Bonn, Germany), Project No. Scha 360/2-2. The calculations were performed at the Rechenzentrum der Universität Kiel.
${ }^{1}$ P. Drahten, W. Ranke, and K. Jacobi, Surf. Sci. 77, L162 (1978).

${ }^{2}$ W. Mönch, in $M B E$ and Heterostructures, edited by L. L. Chang and K. Ploog (Nijhoff, Amsterdam, 1985).

${ }^{3}$ P. J. Dobson, J. H. Neave, and B. A. Joyce, Surf. Sci. 119, L339 (1982).

${ }^{4}$ P. K. Larsen, J. F. van der Veen, A. Mazur, J. Pollmann, J. H. Neave, and B. A. Joyce, Phys. Rev. B 26, 3222 (1982).

${ }^{5}$ P. K. Larsen, J. H. Neave, J. F. van der Veen, P. J. Dobson, and B. A. Joyce, Phys. Rev. B 27, 4966 (1983).

${ }^{6} \mathrm{R}$. D. Bringans and R. Z. Bachrach, in Proceedings of the 17th International Conference on the Physics of Semiconductors, San Francisco, 1984, edited by W. A. Harrison (Freeman, San Francisco, 1984).

${ }^{7}$ L. G. Salmon and T. N. Rhodin, J. Vac. Sci. Technol. B 1, 736 (1983).

${ }^{8}$ P. K. Larsen, J. F. van der Veen, A. Mazur, J. Pollmann, and B. H. Verbeek, Solid State Commun. 40, 459 (1981).

${ }^{9}$ T.-C. Chiang, R. Ludeke, M. Aono, G. Landgren, F. J. Himpsel, and D. E. Eastman, Phys. Rev. B 27, 4770 (1983).

10J. R. Chelikowsky and M. L. Cohen, Phys. Rev. B 14, 556
(1976).

${ }^{11}$ From our photoemission work on $\mathrm{GaAs}(110)$ it results that $\left|V_{0}\right|$ is about $15 \mathrm{eV}$ for the $\Gamma-K-X$ emission direction.

${ }^{12}$ M. Cardona, N. E. Christensen, and G. Fasol, Phys. Rev. B 38, 1806 (1988).

${ }^{13}$ G. P. Williams, F. Cerrina, G. J. Lapeyre, J. R. Anderson, R. J. Smith, and J. Hermanson, Phys. Rev. B 34, 5548 (1986).

${ }^{14}$ J. Henk, W. Schattke, H.-P. Barnscheidt, C. Janowitz, R. Manzke, and M. Skibowski, Phys. Rev. B 39, 13286 (1989).

${ }^{15}$ W. Mönch, in Festkörperprobleme (Advances in Solid State Physics), edited by P. Grosse (Vieweg, Braunschweig, FRG, 1984), Vol. XXIV, p. 229.

${ }^{16}$ R. Hoffmann, J. Chem. Phys. 39, 1397 (1963).

${ }^{17}$ E. Clementi and C. Roetti, At. Data Nucl. Data Tables 14, 177 (1974).

${ }^{18}$ M. L. Cohen and T. K. Bergstresser, Phys. Rev. 141, 789 (1966).

${ }^{19}$ J. R. Chelikowsky, T. J. Wagener, J. H. Weaver, and A. Jin, Phys. Rev. B 40, 9644 (1989).

${ }^{20}$ M. Lehmann, master's thesis, Universität Kiel, 1988. 

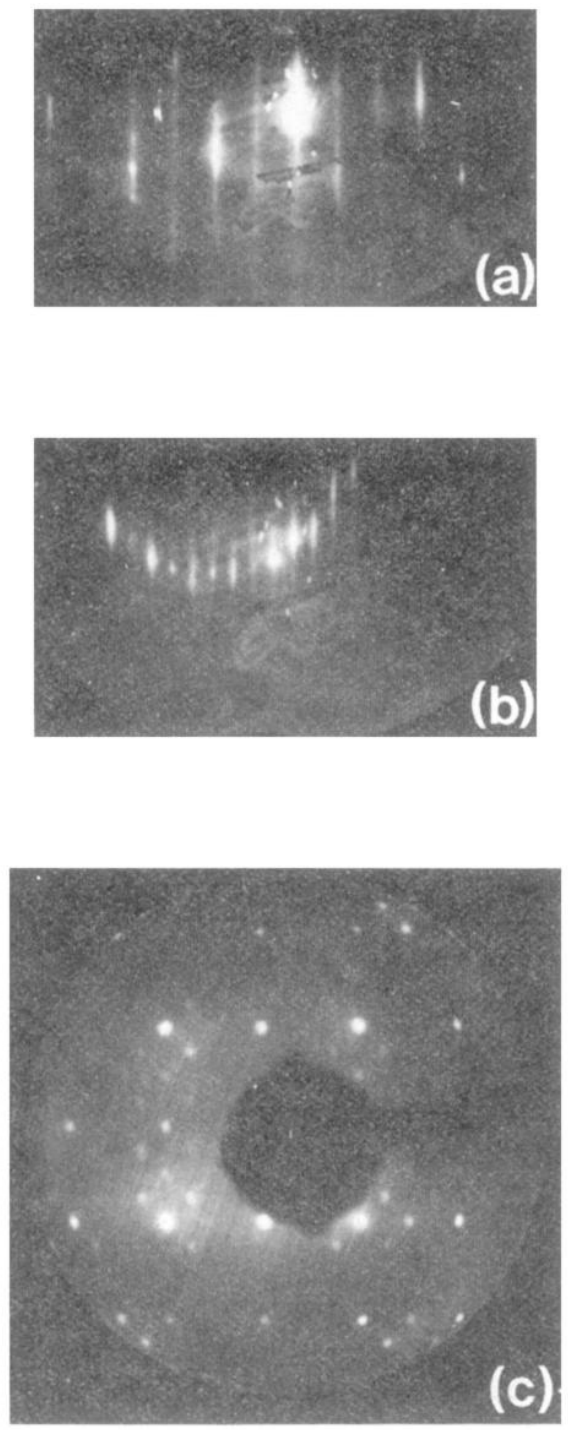

FIG. 1. (a) and (b) RHEED pattern of GaAs(001)- $(2 \times 4)$ during the growth at $535^{\circ} \mathrm{C}$ taken with $5 \mathrm{keV}$ electron energy. In (a), the [110] azimuth is shown. The periodicity is doubled relative to the ideal surface along [ 110$]$, i.e., every second streak is due to the $(1 \times 1)$ surface. (b) is the same for the [110] azimuth: The periodicity is four times that of the ideal $(1 \times 1)$ surface along [110]. The center of the pictures is overexposed due to imperfections in the screen. (c) LEED pattern of $\mathrm{GaAs}(001)$ $c(4 \times 4)$ at room temperature taken with $116 \mathrm{eV}$ electron energy. 